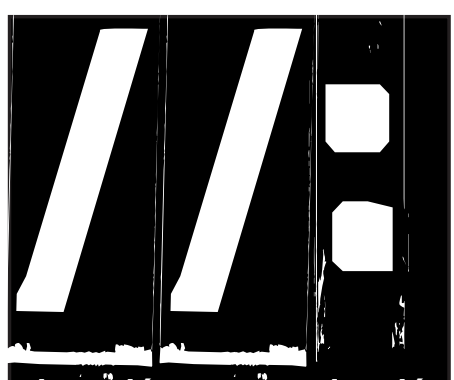

aducación y cömunicación 3: 13-26 Nov. 2011

\title{
Y VIVIERON FELICES COMO REY Y REY. EDUCACIÓN INFANTIL Y HOMOSEXUALIDAD: UN CUENTO INACABADO
}

\section{And They Lived Happily After as King and King. Primary Education and Homosexuality: An Unfinished Story}

\author{
Dr. Alfons Romero \\ Profesor Titular de Sociología. Facultad de Educación y \\ Psicología. Universidad de Girona (España) \\ E-mail: alfons.romero@udg.edu \\ Paco Abril \\ Profesor Asociado. Facultad de Educación y Psicología. \\ Universidad de Girona (España) \\ E-mail: francisco.abril@udg.edu
}

\begin{abstract}
Resumen:
El artículo muestra los resultados españoles del proyecto europeo de investigación Gender Loops sobre la introducción de la homosexualidad en la etapa de Educación Infantil. El artículo se divide en tres partes. En la primera parte se reivindica la necesidad de introducir la educación sobre la homosexualidad en la etapa de educación infantil para su consecuente normalización social. La segunda parte analiza los resultados obtenidos a partir de encuestas y entrevistas en profundidad. La tercera parte describe cómo introducir la homosexualidad en la educación infantil, con ejemplos prácticos, y da cuenta de una experiencia de implementación de este tipo de diversidad en un centro de educación infantil. Los resultados muestran que los centros de formación del profesorado no tienen una estrategia definida para la introducción de esta temática en sus estudios. Y es que los propios centros de formación inicial siguen considerándolo un tema para tratar en etapas educativas posteriores.

Palabras clave: Educación Infantil, Homosexualidad, Diversidad Sexual, Formación del profesorado
\end{abstract}

\begin{abstract}
This article shows the Spanish findings that form part of the European Research programme "Gender Loops" which explores the introduction of homosexuality in primary education. In the first part of the article we shall defend the necessity to introduce the theme of homosexuality within primary education so as promote its social integration. The second part of the article shall analyze the results obtained from surveys and interviews. The third part shall describe how to introduce the theme of homosexuality within the primary educative system, including practical examples of how this initiative has been implemented within a primary school. The findings of our research show that a unified strategy does not exist to introduce this theme with the educative system. What is more, primary schools consider this area as something to be dealt with at higher levels within the educative system. Key words: Primary education, homosexuality, sexual diversity, teacher training.
\end{abstract}

Recibido: 11-07-2011 / Revisado: 31-07-2011 / Aceptado: 9-08-2011 / Publicado: 30-10-2011

https://dx.doi.org/10.25267/Hachetetepe.2011.v2.i3.3 


\section{Introducción}

耳 ste artículo presenta los resultados de la _investigación del proyecto Gender Loops "Metodologías, recursos y estrategias para trabajar el género y la diversidad afectivo-sexual en la educación infantil" financiada por la Unión Europea en el marco del programa Leonardo. El proyecto Gender Loops pretende implementar la perspectiva de género en los centros de formación del profesorado de Educación Infantil, en la formación continua y en los propios centros de Educación Infantil. El proyecto se llevó a cabo entre los años 2007 y 2009. Los países participantes en la investigación son Alemania, Lituania, Noruega, Turquía y España (más información global del proyecto en www.genderloops.eu). El grueso de la investigación se ha centrado en los temas de género, sin embargo el equipo español consideró oportuno incluir preguntas que hicieran referencia a la diversidad afectivo-sexual. Este artículo presenta los resultados específicos españoles sobre esta temática. El artículo se estructura en tres partes. En la primera parte, partiendo de estudios previos a nivel internacional, se reivindica la necesidad de introducir la educación sobre la homosexualidad en la etapa de educación infantil para su consecuente normalización social. La segunda parte analiza los resultados obtenidos a partir de una serie de encuestas y entrevistas en profundidad. Finalmente, la tercera parte, describe cómo introducir la homosexualidad en la educación infantil, con ejemplos prácticos, y da cuenta de una experiencia de implementación de este tipo de diversidad en un centro de educación infantil.

\section{Metodología}

La recogida de datos se ha realizado a partir de tres técnicas de investigación. En un primer momento se realizó un análisis documental de los planes de estudios y materiales en relación a la diversidad afectivosexual. Posteriormente se elaboró un cuestionario que fue enviado a través de Internet a todos los centros de formación universitarios y de formación profesional que imparten en España la titulación de maestro/a o técnico/a en infantil (1). En total se contactó con 63 universidades y 207 centros de secundaria. El cuestionario iba dirigido a la dirección del centro en los casos de secundaria y al decanato de las facultades en el caso de las universidades. Se les pedía que la encuesta fuera respondida por la coordinación de los estudios de infantil o las personas responsables de implementar el género. Se hizo un seguimiento telefónico para reforzar el porcentaje de respuestas y comprobar que los cuestionarios habían llegado a la persona adecuada. El porcentaje final de respuestas ha sido muy elevado, un 30\% del universo. Se obtuvieron 81 cuestionarios, 23 procedentes de universidades y 58 de centros de secundaria, donde se estudia la especialidad de técnico/a de infantil. Finalmente se realizaron 10 entrevistas semi-estructuradas a responsables de la coordinación de los estudios de infantil, alumnos/as y representantes de la Administración Educativa. 


\section{1/. Y viviveron felices como rey y rey. Educación infantil y homosexualidad: un cuento inacabado}

\section{Monográfico}

\section{Parte 1: Punto de partida}

La normalización social de la homosexualidad en el aula

"A pesar de la creencia general en lo contrario, los temas de igualdad

de gays y lesbianas son relevantes para todos/as los/ as niños/as y sus

familias al margen de sus orientaciones sexuales"

(Robinson y Jones díaz, 2006:166)

Si bien no podemos negar la existencia de alumnado, profesorado y padres y madres homosexuales, lesbianas, bisexuales o transgenéricos en nuestras escuelas, seguimos lejos de la normalización social de la diversidad afectivo-sexual en el aula, especialmente en el ámbito de la educación infantil. Toda la educación está pensada para niños/as heterosexuales, de manera que se tratan los deseos, las identidades sexuales, las vivencias y actitudes de los chicos/as como heterosexuales. Este planteamiento es, consecuentemente, discriminatorio y excluyente.

Las discusiones sobre este tema toman un cariz especial en el caso de la educación infantil, donde se argumenta que es un tema que debe tratarse (si debe) en etapas posteriores. Se argumenta a menudo que se trata de un tema no apropiado para tratar en una edad tan temprana, que la familia es el contexto en el que estos temas deberían tratarse con los/as niños/as, etc:

«En nuestra investigación sobre este tema con los/as educadores/as infantiles, la sexualidad, especialmente la referida a la sexualidad de gays y lesbianas- es vista generalmente como un tema de 'adultos' exclusivamente, con la mayoría de los/as educadores/as infantiles considerando que los/as niños/as son demasiado jóvenes para comprender estos temas» (Robinson y Jones Díaz, 2006:151)
Sólo en el caso de la diversidad familiar, se reconoce la necesidad de un cierto tratamiento del tema: la importancia de reconocer las experiencias familiares de los/as niños/as ha sido uno de los elementos que ha hecho surgir el debate en el ámbito de la educación infantil.

Diversos estudios muestran que las tasas de suicidio entre los jóvenes homosexuales son mucho más elevadas que las de los heterosexuales (Halpert, 2002; McDaniel, Purcell y D'Augelli, 2001) (2). No podemos continuar negando la existencia de la diversidad afectivo-sexual en nuestra práctica educativa. No hay razones que justifiquen que los jóvenes homosexuales tengan que vivir su diferencia como un problema, como un proceso traumático porque la escuela les ignora. El sistema educativo debería normalizar esta diversidad afectivo-sexual.

Inspirados por el ejemplo de las minorías étnicas y por las mujeres para incluir la diversidad cultural y de género en las aulas, homosexuales y lesbianas han empezado a reclamar:

- Que las identidades de los homosexuales, lesbianas y transgenéricos sea vista como parte normal de la diversidad de los centros de educación infantil.

- Que se ataque de raíz por parte de los centros de educación infantil el acoso homofóbico que sufren algunos niños/as.

- Un cambio en el currículo para introducir la diversidad afectivo-sexual en todas las áreas.

Se pide, en definitiva, la normalización social de la diversidad afectivo-sexual en el aula. 


\author{
El heterosexismo y la homofobia en la educación \\ infantil
}

Sabemos que las identidades sexuales y de género no vienen dadas biológicamente, sino que son creadas y recreadas a través de prácticas institucionales. En este sentido los centros de educación infantil pueden ser vistos como un lugar de producción de identidades de género y sexo y no sólo como una agencia que de manera pasiva refleja las relaciones de poder dominante. Investigación reciente sobre masculinidad ha contribuido a este debate poniendo de relieve que también los chicos están sometidos a presiones de género y de identidad sexual (Lomas, 2003; Lesko, 2000; Bourdieu, 2000).

La tan extendida idea de que hasta hace poco en la escuela no se trataba el tema de la sexualidad es falsa: la sexualidad ha estado presente siempre. El discurso subyacente sobre la sexualidad que era y continúa siendo proporcionado mayoritariamente por la escuela es la "naturalización" de la heterosexualidad, presentando el resto de variaciones como desviaciones (Epstein, D., 1994; Kehily, M.J., 2002; Lipkin, M. y Ghaill, M., 1996). Esto se ve muy claro en los debates sobre la no necesidad de hablar de sexualidad en los niveles de educación infantil. En el caso de la educación infantil hay un modelo de sexualidad predominante heterosexual que es básico para la hegemonía del heterosexismo, dado que se da durante lo que los sociólogos llamamos primera socialización, que es la más fuertemente enraizada en la conciencia y personalidad de los individuos.

Heterosexismo y homofobia se dan la mano en la etapa de educación infantil. Entendemos por heterosexismo el silencio sobre la heterosexualidad y la presunción de que todo el mundo es heterosexual. Utilizamos el término homofobia para referirnos a los ataques explícitos o no a homosexuales y lesbianas. El heterosexismo acostumbra a actuar en silencio. A menudo se considera que la homofobia implica lo contrario que sea un ataque explícito, pero como parte del heterosexismo, la homofobia también es el silencio y la ausencia. El heterosexismo es homófobo porque no reconoce o discrimina la diferencia:

«La heterosexualidad se convierte en una voz "pública", la definición y la representación de la sexualidad "normal" y "natural". Esta normalización de la heterosexualidad es más cumplir a través de los discursos de género de gran alcance que operan para constituir actuaciones sancionadas por la sociedad de la masculinidad y la feminidad como heterosexuales y regular la conformidad de género entre niños y jóvenes. La educación infantil es una parte muy importante de este proceso de normalización de la construcción del binario heterosexual pública / homosexual privada» (Robinson y Jones, 2006:158).

Esta naturalización de la heterosexualidad tiene otro efecto: su ausencia del currículo.

\section{Parte 2: Resultados de la investigación}

La nueva legislación sobre parejas del mismo sexo en España (ley de matrimonio y adopciones) ha situado el tema de los modelos de familia no tradicionales y la diversidad afectivo-sexual como un asunto a tratar en los estudios de infantil. Muchos de los/las entrevistados/as coinciden en la necesidad de formar al profesorado de infantil en estas, nuevas, realidades. Por otro lado, aunque es un tema aún poco desarrollado 


\section{1/: Y viviveron felices como rey y rey. Educación infantil y homosexualidad: un cuento inacabado}

\section{Monográfico}

y trabajado en los centros, hoy día se hace necesario que el profesorado de infantil sea capaz de gestionar la diversidad afectivo-sexual presente en un aula. En una sociedad abierta y plural es necesario tratar estos temas con naturalidad y formar al profesorado en competencias que le permitan trabajar la riqueza y diversidad de las aulas.

En la encuesta online enviada a los centros de formación inicial, las respuestas sobre si el alumnado sale preparado para tratar la diversidad afectivo-sexual en el aula, obtenían respuestas muy favorables. El 61,6\% cree que el alumnado está muy o bastante capacitado para tratar la diversidad afectivo-sexual (tabla 1).

Tabla 1: Considera que los/as alumnos/as que forman estarán capacitados/as para tratar la diversidad afectivo-sexual

\begin{tabular}{ll}
\hline & $\begin{array}{l}\text { Tratar la Diversidad } \\
\text { afectivo-sexual }\end{array}$ \\
\hline Muy capacitado/a & 10,3 \\
\hline Bastante Capacitado/a & 51,3 \\
\hline Más o menos Capacitado/a & 25,6 \\
\hline Poco capacitado/a & 10,3 \\
\hline Nada capacitado/a & 2,6 \\
\hline Total & 100,0 \\
\hline
\end{tabular}

Estos datos contrastan con la opinión de los/las entrevistados/as, y con los datos de la misma encuesta que señalan algunas carencias formativas respecto a la diversidad afectivo-sexual. Asimismo, en el análisis que se hizo de los planes de estudios de la especialidad de infantil de las universidades españolas así como en las entrevistas realizadas no se han encontrado asignaturas que traten específicamente el tema de la orientación y diversidad afectivo-sexual. Si se trata, se hace a iniciativa de algún/a profesor/a interesado/a en ello o por ser noticia de actualidad.

«Como asignatura no. En la asignatura que se trata los diferentes tipos de familia [Bienestar y Salud] se habla un poco de la diversidad familiar y también de la sexualidad de los niños y niñas (...) En el plan de estudios no consta, pero en el programa de la asignatura [Bienestar y Salud] sí consta»." (Coordinadora universidad estudios educación infantil)

En relación a esto, El gráfico 1 muestra más concretamente qué temas se tratan a nivel global en las asignaturas de los estudios de educación infantil. La diversidad afectivo-sexual ocuparía un lugar intermedio en el gráfico. El 49,4\% de los centros encuestados señalan que tratan o han tratado esta temática. En muchos casos, como luego pudimos comprobar en las entrevistas semi-estructuradas, este tratamiento se limitaba a introducir, en una sesión, la temática de la diversidad familiar, a raíz de la ley de matrimonio y adopción de parejas del mismo sexo aprobada en 2006 en España:

«Relacionado con este tema [de la familia] si que está recogido. Este tema, el año pasado fue uno de los que más salió porque se aprobó el matrimonio homosexual, pues esto se trabajó» (Coordinadora universidad estudios educación infantil) 


\section{II: Homosexualidad e Imagen}

Gráfico 1. En el centro se trata sobre....

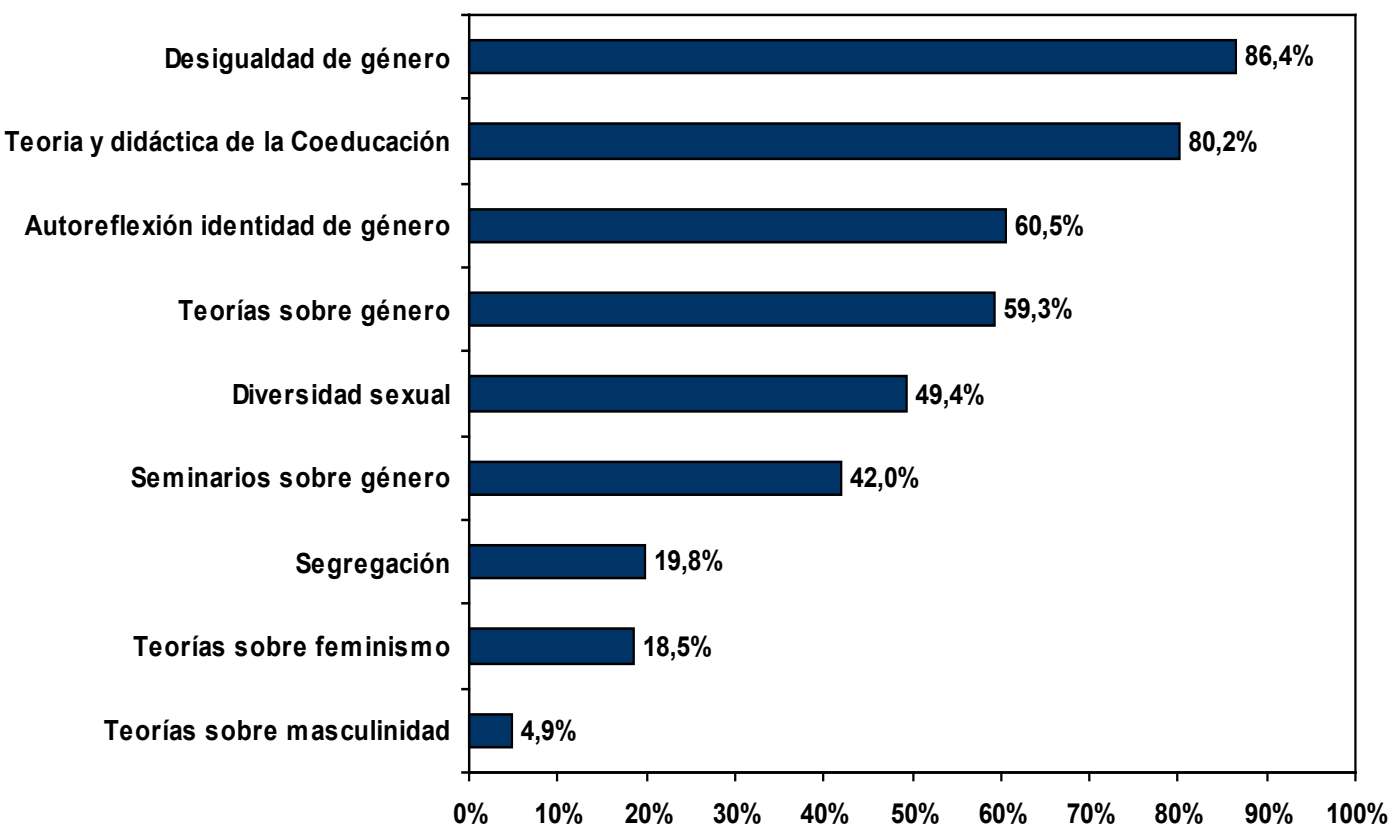

Otro dato interesante es el que hace referencia a la opinión de si la homosexualidad y la diversidad afectivo-sexual deben tratarse en la educación infantil. Casi la mitad de los/as encuestados/as considera que son temas adecuados para tratar en otras etapas educativas y no en infantil (tabla 2). Existe, por tanto, un alto porcentaje de personas (coordinadores/as de los estudios de infantil) que tendrían algún tipo de prejuicio u opinión en contra a la hora de implementar la diversidad afectivo-sexual en la etapa de infantil 
Tabla 2: ¿Considera oportuno tratar los siguientes temas en la etapa de educación infantil?

\begin{tabular}{|l|l|l|l|l|}
\hline & Sí, muy oportuno & $\begin{array}{l}\text { Mejor en otras etapas } \\
\text { educativas } \\
\text { posteriores }\end{array}$ & $\begin{array}{l}\text { No son temas para } \\
\text { tratar en educación }\end{array}$ & Total \\
\hline $\begin{array}{l}\text { Homosexualidad y di- } \\
\text { versidad afectivo-sexual }\end{array}$ & 46,9 & 48,1 & 4,9 & 100 \\
\hline
\end{tabular}

Respecto a los recursos, o material que dispone el centro para tratar temas de diversidad afectivo-sexual (tabla 3). El $64,7 \%$ de los centros de formación inicial no dispone de recursos didácticos sobre diversidad afectivo-sexual.

Tabla 3: El Centro Dispone de Recursos Didácticos sobre:

\begin{tabular}{|l|l|l|}
\hline & Si & No \\
\hline Diversidad afectivo-sexual & 35,3 & 64,7 \\
\hline
\end{tabular}

En cuanto a materiales y metodologías para trabajar la diversidad afectivo-sexual, en los últimos años, gracias a asociaciones GLBT se han elaborado y difundido materiales para trabajar la diversidad y orientación sexual en el aula. Sin embargo, la mayoría de materiales se dirigen a la etapa de primaria (612 años) y secundaria (12-16 años). Hay algunos cuentos dirigido a niños/as más pequeños/as, pero son necesarios más materiales didácticos, audiovisuales y métodos, para trabajar con los/las profesores/as y los/ las niños/as de la etapa infantil

Estos últimos datos contrastan con el hecho de que en un $49,4 \%$ de los centros afirme tratar la diversidad afectivo-sexual en algunas de las asignaturas y con el optimismo con el que se responde a la capacitación de su alumnado para tratar profesionalmente estas cuestiones en el futuro (sólo un 12,9\% de los centros afirma que su alumnado no estará capacitado para tratar sobre diversidad afectivo-sexual). Asimismo pudimos comprobar en las entrevistas al alumnado la poca (in) formación sobre temas de diversidad afectivo-sexual. Por ejemplo en el siguiente comentario de una alumna se observa confusión e ideas equivocadas en torno a los roles en las parejas del mismo sexo.

«Lo estamos trabajando en psicología [el tema de las parejas homosexuales]. Si hay alguien que haga de madre y otro de padre pues no hay ningún problema para el niño» (Alumna 1, Universidad)

Por tanto, actualmente, no se está haciendo todo lo que se tendría que hacer para introducir la diversidad afectivo-sexual en la etapa de la educación infantil. En primer lugar porque el profesorado no está, en general, preparado para introducir estos temas y en segundo lugar porque no existen materiales adecuados para ello, tal como señala esta entrevistada. 
«No se está haciendo, se tendría que hacer. Tenemos mucho trabajo. Quiero ser optimista, creo que el profesor no es que no quiera hacerlo, es que no sabe cómo hacerlo. Nosotros tenemos un cuento para trabajar la diversidad afectivo-sexual pero lo que pasa es que sólo lo trabajan en los centros que tienen programas de coeducación (...) Esto se tendría que introducir paulatinamente» (Responsable Coeducación Administración Autonómica)

\section{Parte 3: Incluyendo la diversidad afectivo-sexual en la educación infantil}

La investigación sobre discriminación racial y sexual ha mostrado que cuanto antes se empiece, más se avanza y más profundo es el grado de igualdad de las minorías. A pesar de ello, es necesaria una programación adecuada a las edades con las que estamos trabajando. Se trata de introducir temas relacionados con la afectividad y el género de manera natural. Las investigaciones también han mostrado cómo la capacidad de darse cuenta de lo que pasa en el mundo y de preguntarse sobre el mundo es especialmente fuerte en los primeros años.

Así, en Challenging lesbian and gay inequalities in education, Akanke (1994) nos pone el ejemplo de una alumna de infantil que le da a su madre la felicitación que ha hecho en clase para el día de la madre. En la felicitación dice "Feliz día de la madre para mi madre y Terry". La madre se espanta y le pregunta a su hija qué ha dicho la maestra al ver el nombre de Terry. La hija, de manera conspiratoria, le dice que ha añadido el nombre en el autobús volviendo hacia casa y que por eso la he escrito con otro rotulador. La separación de espacios, de lenguaje, de actitud... nos muestra el cambio de registro capaz de realizar por cualquier criatura.

\section{Currículo oculto}

El concepto de currículo oculto es utilizado por los científicos sociales para poner de relieve que el aprendizaje sobrepasa las fronteras del currículo oficial y que tiene efectos muy a menudo no apreciados. Los niños/as aprenden también a conformarse o a resistir la cultura oficial de la escuela. Como señalan García y Puigvert (2003) el currículo oculto son todos los aspectos que, sin ser explicitados, ni a menudo debatidos, se transmiten a los y las estudiantes a través de estructuras que subyacen tanto a los contenidos formales, como a las formas de relaciones sociales que se dan en la escuela.

Desde este punto de vista, obviamente, el currículo oculto también puede ser visto en términos de regulación de categorías de sexo y género. Es necesario remarcar, que a pesar de que la educación infantil trabaja de manera transversal el tema de los valores, generalmente no se plantea la inclusión de la erradicación de la homofobia entre sus objetivos. Esto es así, tanto en la programación de la educación en valores, como para la propia transmisión de valores por parte del profesorado.

\section{Propuestas para el Currículo Oculto:}

A continuación detallamos algunas sugerencias para luchar contra la homofobia que se produce en la transmisión del currículum oculto:

- Cada vez que un/a niño/a hace un comentario homofóbico, el profesor/a debería llamar la atención sobre el carácter vejatorio e intolerante del comentario.

- No dirigir siempre al alumnado homosexual o diferente al psicólogo o psicopedagogo del centro (para no hacerlos sentir tan enfermos y extraños). No hacer- 


\section{1/: Y viviveron felices como rey y rey. Educación infantil y homosexualidad: un cuento inacabado}

los sentir como víctimas, etc.

- Cambiar nuestra asunción de que todos/as los/as niños/as son heterosexuales, y actuar, pensar y hablar teniendo en cuenta la existencia de la diversidad afectivo-sexual en el nuestros centros.

En coherencia con este último punto: utilizar un lenguaje no discriminatorio hacia esta diversidad, hablar de padres y madres en plural, de la posibilidad de que los/as hijos/as tengan dos padres o madres, utilizar cónyuge en lugar de esposo o esposa, etc.

- Utilizar un lenguaje en historias, ficción, permitiendo la presencia de homosexuales y lesbianas: “¿Jaime debe tener novio o novia, no?".

- Solicitar la cooperación de asociaciones que trabajen el tema de la diversidad afectivo-sexual y la educación.

- Comunicación y negociación con las familias. Es importante explicar los proyectos, ya que esto puede ayudar a todas las familias (pero especialmente a los padres homosexuales y madres lesbianas) a una mayor implicación en el centro.

\section{Incluyendo la diversidad afectivo-sexual en el cu- rrículum formal}

Introducción del tema de la diversidad afectivo-sexual en los proyectos de centro, con alusión explícita a la lucha contra la homofobia. Así, en la filosofía del centro y para aquellos temas sobre tolerancia, desigualdad, o perspectiva de género se puede hacer referencia explícita al tema de la diversidad afectivosexual. Como señalan Robinson y Jones Diaz, esta introducción no produce resistencia entre las familias: "En nuestra experiencia e investigación en esta área, las instituciones de infantil que de manera abierta e igualitaria incluyen los temas de equidad de gays y lesbianas en los programas de justicia social y equidad no cuentan con la resistencia de los padres y la comunidad que a menudo siguen, como una decisión" (Robinson y Jones Diaz, 2006:164-165).

Empezar por trabajar la diversidad afectivo-sexual a través de la diversidad familiar es uno de los elementos que más se ha trabajado y sobre el que existe más material. Si bien tenemos que tener presente que la igualdad y la lucha contra la homofobia van mucho más allá de trabajar en clase la diversidad familiar.

Es necesario que los/as educadores ayuden a los niños/as a pensar críticamente sobre los discursos normativos de género y diversidad afectivo-sexual. El trabajo sobre la identidad y los estereotipos puede ayudar a la deconstrucción de la heteronormatividad. Los principales temas a través de los cuales hacerlo son los relacionados con la identidad de género y la diversidad familiar.

Trabajar en la dirección de romper con los prejuicios. Así por ejemplo se suele reducir la introducción de las temáticas que afectan a gays y lesbianas con cuestiones sexuales, en vez de hacerlo sobre las relaciones de cariño, afectividad o amor. La homofobia opera así de manera que define y esencializa la no-heterosexualidad estrictamente en terminus de actos físicos sexuales.

Es importante proporcionar a los/as niños/as recursos que les ayuden a deconstruir los estereotipos y mitos que se mantienen sobre gays y lesbianas y que subrayan la discriminación, acoso y violencia que pueden encontrar en sus vidas. Esta discriminación empieza justamente en los primeros años de la vida, cuando los/as niños/as negocian los discursos normativos que operan sobre el género y la sexualidad.

Positivización de la diversidad en la construcción de las identidades de los niños/as: "Por lo general, 
se confirma que la pedagogía de la primera infancia refleja y potencia la diversidad de antecedentes culturales de los niños y las familias con las que se trabajan. Esta representación positiva o la diversidad de identidades de los niños en la programación diaria y la planificación de la educación infantil se considera fundamental para un idóneo desarrollo individual de la "autoestima", así como fomentar su aprecio por la diversidad que existe de manera más amplia en la sociedad" (Robinson y Jones Díaz, 2006:147).

\section{Una experiencia práctica: hay príncipes que se enamoran de príncipes}

\section{Profesor (leyendo el cuento Rey y Rey):}

"Fue un amor a primera vista príncipe tan guapo

(a los niños/as) ¿Sabéis que ha pasado?

Niños/as: Si, se han enamorado

Niño: Son gays.

(...)

Profesor (leyendo el cuento):

"Se casaron y vivieron felices como Rey y Rey.

Y la reina por fin pudo descansar"

(a los niños/as) ¿Os ha gustado?

Niña: A mi no....Yo quiero que se case con la princesa, es muy guapa

Niño: ...Yo fui a una boda que se casaban dos mujeres...

( ... Sigue la conversación en torno a como fue la boda que se casaban dos lesbianas amigas de sus padres...) Esta transcripción, de una de las sesiones en las que se introdujo la diversidad afectivo-sexual a partir de la presentación de un cuento, muestra las posibles respuestas que nos podemos encontrar. Por un lado la normalidad con la que algunos/as niños/as viven este tema. Por otro las resistencias y la homofobia aprendida (en la familia, los medios de comunicación y la propia escuela) con la que nos podemos encontrar. Precisamente para evitar que la homofobia se instale y asiente, es necesario trabajar con metodologías que normalicen y visibilicen la diversidad afectivo-sexual en la sociedad.

La introducción de la diversidad afectivo-sexual en la etapa de educación infantil era, a priori, uno de los temas complicados del proyecto Gender Loops. Por un lado porque hay pocas experiencias y materiales adecuados a la etapa. Por otro lado, por los miedos y el posible rechazo de los/las niños/as, profesorado y familias.

El profesorado aceptó realizar esta experiencia. Eran consientes de que en sus centros "same sex parents" serán cada vez más visibles y que no están preparados para abordar el tema.

A continuación describimos el proceso de implementación de esta experiencia que se realizó en cuatro centros de educación Infantil, en los niveles de 4 y 5 años.

El primer paso fue analizar y localizar materiales para trabajar la homosexualidad en la etapa de infantil. A pesar que empieza haber materiales, estos aún no están pensados para esta etapa. Lo que si encontramos fue un considerable número de cuentos (3). La mayoría de los cuentos hacían referencia a niños/as que tienen padres del mismo sexo. Todavía hay poco material que aborde la diversidad afectivo-sexual en niños y niñas.

Se eligió el cuento "Rey y Rey" (De Haan y Nijland, 2004) porque era un libro sencillo para que lo entendieran niños y niñas, con una estructura clásica pero un desarrollo y final sorprendente. El cuento aborda la orientación sexual con naturalidad, partiendo de la idea de una Reina que quiere que su hijo príncipe se 


\section{1/: Y viviveron felices como rey y rey. Educación infantil y homosexualidad: un cuento inacabado}

\section{Monográfico}

case para que ella pueda jubilarse. La madre le organiza encuentros con princesas de lugares lejanos, pero ninguna le gusta al príncipe. Hasta que al final se enamora del hermano de una princesa que le han presentado. Los dos príncipes se casan, convirtiéndose en Rey y Rey, mientras que la vieja Reina puede por fin descansar.

El cuento se escaneó y se presentó en una proyección mientras se explicaba. Esta forma de presentación posibilita que niños y niñas estén más concentrados en la historia. También facilita el debate y los comentarios sobre los dibujos y contenidos que aparecen en la pantalla. Durante la exposición se permite que los niños/ as comenten o se les hace preguntas en relación a los dibujos o la historia que se está presentando. En este punto pueden aparecer resistencias e incluso homofobia a la historia que se cuenta. En los centros donde implementamos este método encontramos algunas de estas reacciones. Un niño dijo "qué asco" cuando en la escena final del cuento los príncipes se besan en la boca. Otra niña no entendía que el príncipe no se casará con la princesa, con lo guapa que era.

Una vez finalizada la presentación del cuento se pide a niños y niñas que dibujen en una hoja aquello que más le ha gustado o interesado. Mientras dibujan el/la profesor/a puede comentar o clarificar algún aspecto de la historia. A la hora de dibujar también pudimos observar cierto rechazo y resistencia. Había, especialmente niños, que no querían dibujar a los dos príncipes juntos, dibujaban solo uno de ellos. Otros/as cambiaban la historia y al lado del príncipe colocaban a la princesa o dibujaban aspectos de menor importancia como la Reina, el paje, la gata, etc. También hubo niños/as, en este caso más niñas, que seguían la historia con fidelidad y dibujaban a los dos príncipes, el pastel de boda,... Los dibujos terminados se pegan en la pared. Es el momento, también, para aclarar dudas y hablar de la orientación sexual.

\section{Conclusiones}

Respecto a la diversidad afectivo-sexual el panorama es aún más desalentador. Los centros tampoco tienen una estrategia definida para introducir esta temática en sus estudios, hay muy pocas asignaturas o profesorado que la incluya y apenas hay materiales y metodologías de trabajo. A pesar de esto, los centros son conscientes que los futuros/as profesores/as de infantil han de tener competencias para trabajar estos temas. Las familias no tradicionales, como las formadas por parejas del mismo sexo, están dibujando nuevas realidades presentes hoy en día en los centros de infantil que los/as maestros/as deben gestionar. Aún queda más camino por recorrer para que desde la formación inicial se traten las identidades sexuales minoritarias, como la homosexualidad o transexualidad, más allá de la problematización que puede suponer para las familias y los niños/as. A pesar que la encuesta realizada señala que la mayoría del alumnado que sale de las universidades y centros de formación profesional preparado para tratar la diversidad afectivo-sexual, otros datos señalan que esto no es así. Hay, todavía, un porcentaje elevado de centros que piensan que la diversidad afectivo-sexual no es un tema que se deba tratar en la etapa de la educación infantil. Teniendo en cuenta que apenas hay materiales y metodologías y esta temática está muy poco introducida en las asignaturas y en los estudios. Con este panorama es difícil que el alumnado salga preparado para tratar temáticas relativas a la homosexualidad en las aulas. 


\section{II: Homosexualidad e Imagen}

Notas

(1) En España existen dos modalidades de profesores en Educación Infantil. El técnico superior en educación infantil y el profesor especializado en educación infantil. El título de Técnico Superior en Educación Infantil se obtiene a partir de la realización de un ciclo superior de formación profesional, al que se puede acceder al finalizar la educación secundaria obligatoria. La especialidad de Maestro en Educación Infantil se estudia en facultades o escuelas universitarias y tiene una duración de cuatro años en los nuevos cursos de grado.

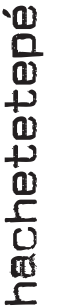

(2) Como evidencia Halpert, S. (2002) estas mayores tasas de suicidio no se deben en si a la orientación sexual, sino a la homofobia y prejuicio que estos adolescentes sufren.

(3) El listado de cuentos sobre diversidad afectivosexual se encuentra en la página web del proyecto: www.genderloops.eu

\section{Referencias}

ACGIL (2000). Educació, homosexualitat i cristianisme. Recursos didàctics per a educadors i educadores. Barcelona: Associació Cristiana de Gais i Lesbianes. Akanke (1994). Black in the Closet, en Epstein, Debbie (Ed.) Challenging Lesbian and Gay Inequalities in Education. Buckingham: Open University Press.

Alonso, Julián; Burgos, Valentín y otros (2002). El respecto a la diferencia por orientación sexual: homosexualidad y lesbianismo en el aula. Oviedo: Xente Gai Astur.

Associació de Pares i Mares de Gais i Lesbianes \& Grup Jove de la CGL, Com dir-ho als pares, Barcelona: Coordinadora Gai Lesbiana.

Boesche, S. (1986). La Martona viu amb l'Eric i en Martí. Barcelona: Institut Lambda.

Borrillo, D. (2001). Homofobia. Barcelona: Bellaterra.

Bourdieu, P. (2000). La dominación masculina. Barcelona: Anagrama.

Cabrerizo, P. y otros. (2000). Construcción del yo, en Payà, Montserrat (Coord) Guía Praxis para el profesorado de ESO. Ética y educación en valores. Barcelona: Cisspraxis.

Cahill, B. \& Theilheimer, R. (1999). Stonewall in the housekeeping area: gay and lesbian issues in the early childhood classroom, en Letts, W.J. \& Sears, J.T. (Eds) Queering Elementary Education: Advancing the Dialogue about Sexualities and Schooling. Lanham, MD: Rowman and Littlefield.

Casper, V.; Cuffaro, H.K.; Schultz, S.; Silin, J.G.; Wickens, E. (1998). Towards a most thorough understanding of the world: sexual orientation and early childhood education, en Yelland, N. (Ed.) Gender in Early Childhood. London: Routledge. 


\section{1/: Y viviveron felices como rey y rey. Educación infantil y homosexualidad: un cuento inacabado}

\section{Monográfico}

Clauzard, Philippe (2002). Conversations sur l'homo(phobie) - L'éducation comme rempart contre l'exclusion. Paris : L'Harmattan.

De Haan, L. y Nijland, S. (2004). Rey y rey. Barcelona: Serres.

Dyer, R. (1993). The Matter of Images: Essays on Representation. London: Routledge.

Epstein, D. (1994). Lesbian and Gay Equality in Education - Problems and Possibilities, en Epstein, D.

(Ed.) Challenging Lesbian and Gay Inequalities in Education. Buckingham: Open University Press.

Epstein, D. y Johnson, R. (1994). On the Straight and the Narrow: The Heterosexual Presumption, Homophobias and Schools, en Epstein, D. (Ed.) Challenging Lesbian and Gay Inequalities in Education. Buckingham: Open University Press.

Escalas, J. y Nolla, E. (2001). Entender a los que entienden: homosexualidad y familia. Barcelona: Juventud.

García, C. y Puigvert, L. (2003). Sociología y currículo, en Fernández Palomares, F. (Coord.) Sociología de la Educación. Madrid: Pearson Educación.

Griffin, Pat (1995). Homophobia in Sport, en Unks, Gerald (Ed.) The Gay Teen. London: Routledge.

Guasch, Ò. (Coord.) (2002). Sociologia de la Sexualitat. Barcelona: Pòrtic.

Halpert, S.C. (2002). Suicidal behavior among gay male youth, en Journal of Gay and Lesbian Psychotherapy, (6), 3: 53-79.

Herat, G. y Koff, Br. (2002). Gestión familiar de la homosexualidad. Barcelona: Bellaterra.

Kehily, M. J. (2002). Sexuality, Gender and Schooling. Shifting Agendas in Social Learning. London: Routledge.

Lesko, N. (Ed) (2000). Masculinities at School. London: Sage Pub.
Lipkin, A. (1995). The case for a Gay and Lesbian Curriculum, en Unks, Gerald (Ed.) The Gay Teen. London: Routledge.

Lipkin, A. (1999). Understanding Homosexuality, Changing Schools. Boulder: Westview Press.

Lomas, C. (2003). ¿Todos los hombres son iguales? Identidades masculinas y cambios sociales. Barcelona: Paidós.

Mac and Ghaill, M. (1994). (In)Visibility: Sexuality, Race and Masculinity in the School Context, en Epstein, Debbie (Ed.) Challenging Lesbian and Gay Inequalities in Education. Buckingham: Open University Press.

Mac and Ghaill, M. (1996). Deconstructing heterosexualities within school arenas, en Curriculum Studies, 4 (2):191-209.

McDaniel J.S., Purcell D.W., D’Augelli A.R. (2001). The relationship between sexual orientation and risk for suicide: research findings and future directions for research and prevention, en Suicide and Life-Threatening Behavior, 31: 84-105.

McLaren, P. (1995). Moral Panic, Schooling, and Gay Identity, en Unks, Gerald (Ed.) The Gay Teen. London: Routledge.

Morrison LL., L'Heureux J. (2001). Suicide and gay / lesbian / bisexual youth: implications for clinicians, en Journal of Adolescence, (24), 1: 39-49.

Newman, L. (2003). Paula tiene dos mamás. Barcelona: Bellaterra.

Núñez, E. (2002). Transsexualisme, transgenerisme i sistemes de gènere, en Guasch, O. (Coord.) Sociologia de la Sexualitat. Barcelona: Pòrtic.

O'Conor, A. (1995). "Who Gets Called Queer in School?”, en Unks, G. (Ed.) The Gay Teen. London: Routledge.

Patrick, P. y Sanders, S. A.L. (1994). Lesbian and Gay 


\section{II: Homosexualidad e Imagen}

Issues in the Curriculum, en Epstein, D. (Ed.) Challenging Lesbian and Gay Inequalities in Education. Buckingham: Open University Press.

Recio, C. (2001). El príncep enamorat. Barcelona: Llibres de l'Índex.

Riesenfeld, R. (2000). Papá, mamá, soy gay. México: Grijalbo.

Robinson, K.H. (2002). Making the invisible visible: gay and lesbian issues in early childhood education, Contemporary Issues in Early Childhood, 3(3):415434.

Robinson, K.H.; Jones Díaz, C. (2006). Diversity and
Difference in Early Childhood Education. Issues for Theory and Practice. Berkshire: Open University Press.

Rofes, E. (1995). Making our Schools Safe for Sissies, en Unks, G. (Ed.) The Gay Teen. London: Routledge. Talburt, S. y Steinberg, Sh. (2005). Pensando queer. Sexualidad, cultura y educación. Barcelona: Graó. Tofiño, I. (Coord, 2004). Gent com tu? Adolescència $i$ diversitat sexual. Barcelona: Viena Edicions.

Uribe, V. (1995). "Project 10. A School-Based Outreach to Gay and Lesbian Youth", en Unks, G. (Ed.) The Gay Teen. London: Routledge. 